\title{
Bulk viscosity effects on elliptic flow
}

\author{
G. S. Denicol ${ }^{a}$, T. Kodama ${ }^{b}$, T. Koide ${ }^{c}$, and Ph. Mota ${ }^{b}$ \\ ${ }^{a}$ Institute für Theoretische Physik, Johann Wolfgang Goethe-Universität, Max-von-Laue Str. 1, 60438, Frankfurt am \\ Main, Germany \\ ${ }^{b}$ Instituto de Física, Universidade Federal do Rio de Janeiro, C. P. 68528, 21945-970, Rio de Janeiro, Brasil \\ ${ }^{c}$ FIAS, Johann Wolfgang Goethe-Universität, Ruth-Moufang Str. 1, 60438, Frankfurt am Main, Germany
}

\begin{abstract}
The effects of bulk viscosity on the elliptic flow $v_{2}$ are studied using realistic equation of state and realistic transport coefficients. We find that the bulk viscosity acts in a non trivial manner on $v_{2}$. At low $p_{T}$, the reduction of $v_{2}$ is even more effective compared to the case of shear viscosity, whereas at high $p_{T}$, an enhancement of $v_{2}$ compared to the ideal case is observed. We argue that this is caused by the competition of the critical behavior of the equation of state and the transport coefficients.
\end{abstract}

\section{Introduction}

The effect of shear viscosity in heavy ion collisions has been studied by several authors [1] and was found to affect considerably the elliptic flow observable $v_{2}$. On the other hand, the bulk viscosity has always been neglected and its effects are mostly unknown. The shear viscosity acts as the resistance against the deformation of a fluid element. In this sense, it is natural to expect that $v_{2}$, which characterizes the spatial anisotropy of the dynamics, is affected by the shear viscosity. On the other hand, the bulk viscosity acts only against the expansion or compression of the fluid. However, this does not mean that the bulk viscosity has negligible effects on $v_{2}$, particularly because the expansion and compression of the produced matter in the collisions is also anisotropic.

Therefore, the behavior of the bulk viscosity coefficient, $\zeta$, near the QCD phase transition should also be carefully studied. Recently, lattice QCD simulations [2] suggest that $\zeta$ is strongly enhanced near the critical temperature $T_{c}$. A similar enhancement will appear even below $T_{c}$, as is shown in [3], where $\zeta$ in the hadronic phase increases also quickly towards $T_{c}$. Considering the fact that the fluid dynamics slows down near $T_{c}$ due to the small sound velocity $c_{s}$, the critical behavior of $\zeta$ should have a crucial role on the expansion of the matter.

In this work, we investigate the effect of bulk viscosity on $v_{2}$ by incorporating the critical behavior of the equation of state (EoS) and $\zeta$ within a $2+1$ dimensional hydrodynamic modeling. On the other hand, the dissipative corrections to the one-particle distribution function at freeze out is not considered, because Grad's method is not applicable for the freeze out values of bulk viscosity obtained in our calculations [4]. 


\section{Relativistic Dissipative Hydrodynamics}

The formulation of relativistic dissipative hydrodynamics is not trivial. The naive relativistic generalization of the Navier-Stokes equation violates causality and leads to intrinsic instabilities making the theory not applicable. In this work, we use the memory function method [5, 6], which is formulated in such a way that the magnitude of the bulk viscosity has a lower bound, and it is possible to implement stable numerical simulations even for ultra-relativistic initial conditions.

We consider the case of vanishing baryon chemical potential where only the conservation of the energy and momentum is required. For a general metric $g_{\mu \nu}$, the continuity equation for the energy-momentum tensor is given by

$$
\frac{1}{\sqrt{-g}} \partial_{\mu}\left(\sqrt{-g} T^{\mu \nu}\right)+\Gamma_{\lambda \mu}^{v} T^{\lambda \mu}=0,
$$

where $\Gamma_{\mu \lambda}^{v}$ is the Christoffel symbol and $g$ is the determinant of $g_{\mu v}$. We use the Landau definition for the local rest frame and assume, as usual, that the thermodynamic relations are valid in this frame. We further ignore the shear viscosity. Then the energy-momentum tensor is expressed as $T^{\mu v}=(\varepsilon+p+\Pi) u^{\mu} u^{v}-(p+\Pi) g^{\mu v}$, where $\varepsilon, p, u^{\mu}$ and $\Pi$ are the energy density, pressure, four velocity and bulk viscosity, respectively. The equation for the entropy production is,

$$
\frac{1}{\sqrt{-g}} \partial_{\mu}\left(\sqrt{-g} s u^{\mu}\right)=-\frac{\Pi}{T} \frac{1}{\sqrt{-g}} \partial_{\mu}\left(\sqrt{-g} u^{\mu}\right) .
$$

where $s$ is the entropy density. From this, we can define the thermodynamic force $F$ associated with the bulk viscosity as $\sqrt{-g}^{-1} \partial_{\mu}\left(\sqrt{-g} u^{\mu}\right)$. It is also useful to introduce the specific volume $\sigma$ defined by the conservation law $\sqrt{-g}^{-1} \partial_{\mu}\left(\sqrt{-g} \sigma u^{\mu}\right)=0$.

In the memory function method, an irreversible current $J$ is induced by the corresponding thermodynamic force as

$$
\frac{J(\tau)}{\sigma(\tau)}=-\int_{\tau_{0}}^{\tau} d \tau^{\prime} \frac{1}{\tau_{R}\left(\tau^{\prime}\right)} \exp \left(-\int_{\tau^{\prime}}^{\tau} \frac{d \tau^{\prime \prime}}{\tau_{R}\left(\tau^{\prime \prime}\right)}\right) \frac{F\left(\tau^{\prime}\right)}{\sigma\left(\tau^{\prime}\right)}+\frac{J\left(\tau_{0}\right)}{\sigma\left(\tau_{0}\right)} \exp \left(-\int_{\tau_{0}}^{\tau} \frac{d \tau^{\prime}}{\tau_{R}\left(\tau^{\prime}\right)}\right) .
$$

Thus, the equation of the bulk viscosity is obtained by setting $J=\Pi$ and $F=\sqrt{-g}^{-1} \partial_{\mu}\left(\sqrt{-g} u^{\mu}\right)$. This is re-expressed in a differential form as follows,

$$
\tau_{\mathrm{R}} u^{\mu} \partial_{\mu}\left(\frac{\Pi}{\sigma}\right)+\frac{\Pi}{\sigma}=-\frac{\zeta}{\sigma} \frac{1}{\sqrt{-g}} \partial_{\mu}\left(\sqrt{-g} u^{\mu}\right),
$$

where $\tau_{\mathrm{R}}$ is the relaxation time and $\zeta$ is the bulk viscosity coefficient.

In the following calculations, we consider the hyperbolic coordinate system $x^{\mu}=\left(\tau, \mathbf{r}_{T}, \eta\right)$, where $\tau=\sqrt{t^{2}-z^{2}}, \quad \eta=\frac{1}{2} \tanh \left(\frac{t+z}{t-z}\right), \quad \mathbf{r}_{T}=(x, y)$ and $\sqrt{-g}=\tau$.

To solve numerically the relativistic hydrodynamic equations (1), (2) and (4), we use the Smoothed Particle Hydrodynamic method [4, 6, 7]. The initial condition used is a parametrization of the usual Glauber model. The form of this initial condition and the choice of parameters can be found in [4]. The impact parameter $b$ is taken to be $7 \mathrm{fm}$. For the EoS, we consider a smooth interpolation between the lattice QCD results [8] and the hadron resonance gas, which is shown in Fig. 1 .

For the bulk viscosity coefficient, we use the result from lattice QCD [2] for the QGP phase and the result from [3] for the hadron phase. Then, the ratio $\zeta / s$ exhibits a maximum at $T_{c}$ and 

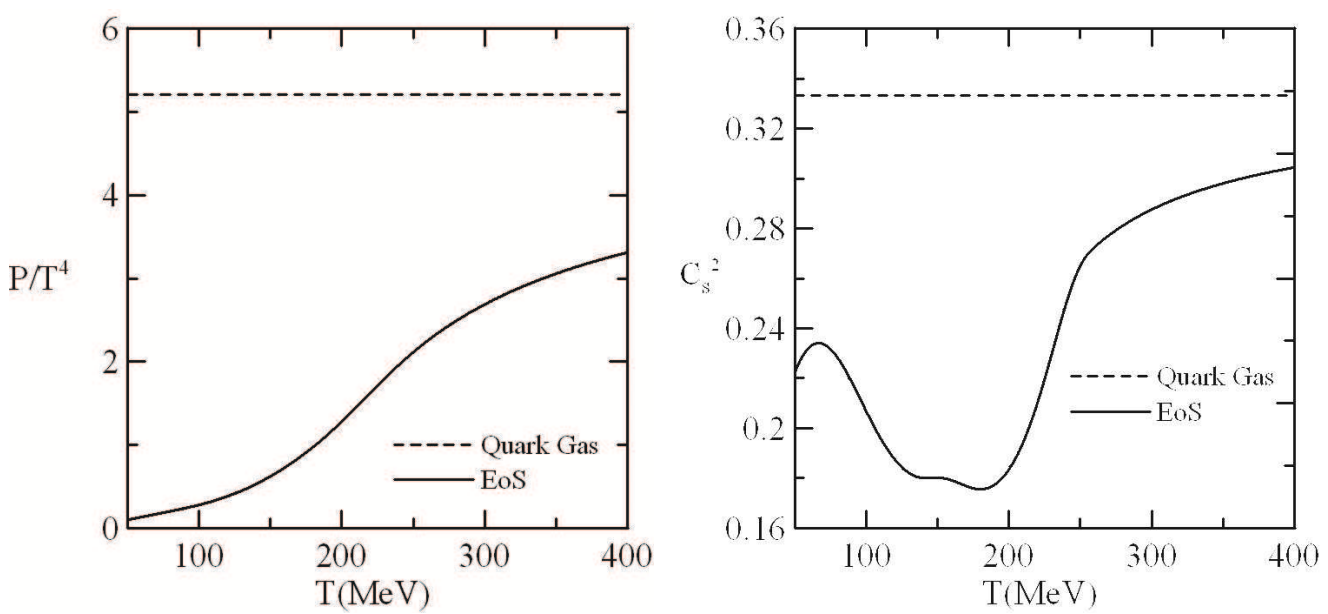

Figure 1: The temperature dependence of the EoS. The solid line corresponds to the EoS used in our calculations. The dotted line denotes the ideal gas of massless quarks EoS.

starts to decrease almost exponentially as the system departs from $T_{c}$. We express these features of $\zeta / s$ as

$$
\underline{\zeta}= \begin{cases}A_{1} x^{2}+A_{2} x-A_{3} & \left(0.995 T_{c} \geq T \geq 1.05 T_{c}\right) \\ \lambda_{1} \exp \left(-(x-1) / \sigma_{1}\right)+\lambda_{2} \exp \left(-(x-1) / \sigma_{2}\right)+0.001 & \left(T>1.05 T_{C}\right) \\ \lambda_{3} \exp \left((x-1) / \sigma_{3}\right)+\lambda_{4} \exp \left((x-1) / \sigma_{4}\right)+0.03 & \left(T<0.995 T_{C}\right)\end{cases}
$$

where $x=T / T_{C}$. The fitted parameters are $\lambda_{1}=\lambda_{3}=0.9, \lambda_{2}=0.25, \lambda_{4}=0.22, \sigma_{1}=10 \sigma_{3}=$ $0.025, \sigma_{4}=0.022, A_{1}=-13.77, A_{2}=27.55$ and $A_{3}=13.45$. For the relaxation time, we use the following parameterization $\tau_{R}=b_{\Pi} \zeta /(\epsilon+p)[6]$. In this work, we use $b_{\Pi}=6$ [4].

\section{Numerical results}

We calculate the elliptic flow parameter, $v_{2}=\langle\cos (2 \phi)\rangle$, for pions as a function of the transverse momentum, using the Cooper-Frye method with a freeze out temperature $130 \mathrm{MeV}$ [4]. In Fig. 2, $v_{2}$ is shown for viscous fluids (dashed, dash-dotted and dotted lines) and ideal fluid (solid line). Dashed and dash-dotted lines correspond to the constant $\zeta / s$ cases and are shown just for the sake of comparison, whereas the dotted line represents the $\zeta / s$ with the QCD phase transition.

For small transverse momenta $P_{T}, v_{2}$ is suppressed by the effect of bulk viscosity, similar to the case of shear viscosity. However, at high $P_{T}, v_{2}$ starts to recover and even surpasses the values obtained from ideal hydrodynamics. This effect is enhanced when $\zeta / s$ exhibits a critical behavior. This behavior at high $P_{T}$ is not observed for the case of shear viscosity and we further confirmed that this is not observed when an ideal gas of massless quarks EoS is used [4]. Thus, this effect should be attributed to the presence of the QCD phase transition, both in the EoS and in the bulk viscosity.

This behavior can be understood as follows. For the viscous case, the radial dependence of the fluid flow is not monotonic in the region where the velocity of sound is minimum. This is because the flow of the internal matter, which has a higher temperature, tends to catch up 


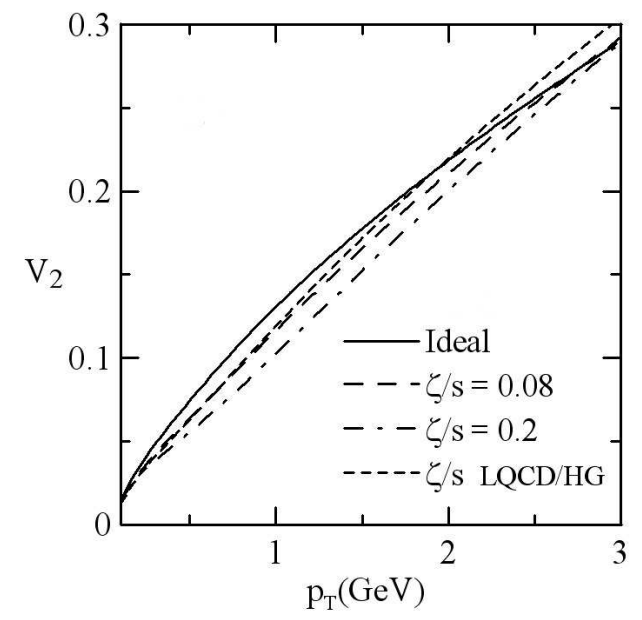

Figure 2: $v_{2}$ as a function of $p_{T}$. The figure displays results for different choices of $\zeta / s$. The solid line corresponds to the ideal result, the dashed and dash-dotted lines correspond respectively to $\zeta / s=0.08$ and 0.2 , and the dotted line is the result for $\zeta / s$ with critical behavior.

the foregoing fluid elements, generating an inhomogeneous velocity field in the radial direction. Then, the bulk viscosity $\Pi$ starts to increase in this region, heating the matter. If this happens, the gradient of $\Pi$ becomes dominant in the acceleration of the fluid compared to the pressure gradient (note that the acceleration is given by $-\nabla(p+\Pi))$ since the gradient of the pressure is proportional to the sound velocity. Such a mechanism works more efficiently in the direction where the initial acceleration is large, and in consequence, in the direction to increase the elliptic flow. Furthermore, this effect becomes effective only when significant collective flow is formed near $\mathrm{T}_{c}$. Thus the recovery of $v_{2}$ by this mechanism is expected for higher momentum particles.

We acknowledge illuminating discussions with T. Hirano and A. Monnai. This work has been supported by CNPq, FAPERJ, CAPES, PRONEX and the Helmholtz International Center for FAIR within the framework of the LOEWE program (Landesoffensive zur Entwicklung Wissenschaftlich- Okonomischer Exzellenz) launched by the State of Hesse.

\section{References}

[1] See, for example, the contribution from P. Romatschke in this volume.

[2] F. Karsch, D. Kharzeev and K. Tuchin, Phys. Lett. B663, 217-221 (2008).

[3] J. Noronha-Hostler, J. Noronha and C. Greiner, arXiv:0811.1571 (2008)

[4] G. S. Denicol, T. Kodama, T. Koide and Ph. Mota, arXiv:0903.3595 (2009).

[5] T. Koide, G. Denicol, Ph. Mota and T. Kodama, Phys. Rev. C75, 034909 (2007)

[6] G. S. Denicol, T. Kodama, T. Koide and Ph. Mota, J. Phys. G35, 115102 (2008).

[7] G. S. Denicol, T. Kodama, T. Koide and Ph. Mota, Phys. Rev. C78, 034901 (2008).

[8] Y. Aoki et al (WHOT-QCD Collaboration), arXiv:0810.4742 (2008). 